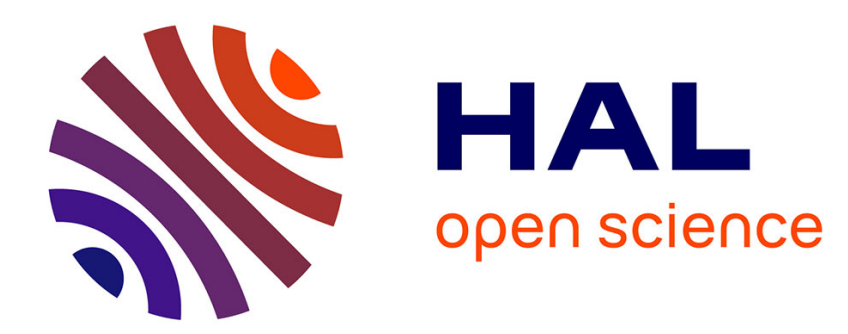

\title{
Zwei oder drei Dinge, die ich von der Technikphilosophie weiss \\ Wolfgang Pircher
}

\section{To cite this version:}

Wolfgang Pircher. Zwei oder drei Dinge, die ich von der Technikphilosophie weiss. Revue de Synthèse, 2009, 130 (1), pp.133-146. 10.1007/s11873-009-0066-7 . hal-00478456

\section{HAL Id: hal-00478456 \\ https://hal.science/hal-00478456}

Submitted on 30 Apr 2010

HAL is a multi-disciplinary open access archive for the deposit and dissemination of scientific research documents, whether they are published or not. The documents may come from teaching and research institutions in France or abroad, or from public or private research centers.
L'archive ouverte pluridisciplinaire HAL, est destinée au dépôt et à la diffusion de documents scientifiques de niveau recherche, publiés ou non, émanant des établissements d'enseignement et de recherche français ou étrangers, des laboratoires publics ou privés. 


\title{
ZWEI ODER DREI DINGE, DIE ICH VON DER TECHNIKPHILOSOPHIE WEISS Eine sehr subjektive Sicht
}

\author{
Wolfgang PIRCHER*
}

\begin{abstract}
Zusammenfassung: Im Schatten der „offiziellen“ Technikphilosophie sind in der jüngeren Vergangenheit Arbeiten entstanden, welche sich verschiedenen Techniken zuwenden und deren konkrete geschichtliche Entwicklung philosophisch aufarbeiten. Nach einem kurzen Abriß der Technikphilosophie in Deutschland seit dem Ende des 19. Jahrhunderts, wird auf eine kleine Auswahl solcher Arbeiten näher eingegangen. Hierbei stellt man eine überraschende Nähe zu den Literatur- und Kulturwissenschaften fest.
\end{abstract}

STICHWÖRTER: Technikphilosophie, Informationstheorie, Wirtschaftsgeschichte, Aufschreibesysteme, Kybernetik.

\section{DEUX OU TROIS CHOSES QUE JE SAIS DE LA PHILOSOPHIE DE LA TECHNIQUE Une vue très subjective}

Résumé: Récemment en langue allemande, sous l'ombre portée d'une philosophie de la technique pour ainsi dire " officielle» sont parus des travaux qui traitent de techniques différentes et qui examinent à nouveaux frais leur développement historique concret du point de vue philosophique. Une fois tracée une brève esquisse de la philosophie de la technique en Allemagne depuis la fin du XIXe siècle, on considère de plus près un échantillon de ces travaux récents. On en vient à constater leur surprenante proximité avec des recherches d'histoire culturelle ou littéraire.

Mots-CLÉs: philosophie des techniques, théorie de l'information, histoire économique, réseaux discursifs, cybernétique.

\section{TWO OR THREE THINGS I KNOW ABOUT PHILOSOPHY OF TECHNIQUE A quite subjective view}

ABSTRACT: Nearby a somehow "official" philosophy of technique, works have appeared in recent German literature where different techniques are studied according a renewed philosophical agenda about their concrete historical development. Once $19^{\text {th }}$ Century German philosophy of technique briefly sketched, a sample from this recent production is considered. Surprisingly, they are meeting current literary studies and cultural history.

KEYWORDS: philosophy of technique, theory of information, economical history, discourse networks, cybernetics.

*Wolfgang Pircher, né en 1946, est Assistenzprofessor à l'Institut für Philosophie de l'université de Vienne. Ses travaux portent sur la philosophie des sciences et des techniques et sur la philosophie politique. Il a notamment dirigé et publié Gegen den Ausnahmezustand. Zur Kritik an Carl Schmitt (Vienne, Springer, 1999), Tyrannis und Verführung (Vienne, Turia und Kant, 2000), Sozialmaschine Geld (Francfort/Main, Anabas, 2000, 2 vol.), Kunst, Zeichen, Technik: Philosophie am Grund der Medien (Münster, Lit, 2004).

Adresse: Institut für Philosophie, Fakultät für Philosophie und Bildungswissenschaft, Universität Wien, Universitätsstraße 7, A-1010 Wien.

Courrier électronique: wolfgang.pircher@univie.ac.at. 
La philosophie des techniques en langue allemande s'est considérablement renouvelée depuis déjà deux ou trois décennies alors qu'en France, pendant la même période, on s'est commodément enferré dans le commentaire, ici, de Habermas et de Heidegger, ou dans le simulacre, là, des études de techniques publiées dans les années 1970 et 1980 en langue anglaise, ignorant cette fois ce que leur généalogie intellectuelle devaient aux sciences sociales et à la philosophie continentale, voire aux misères de leur circulation internationale.

À ce jour aucun ouvrage personnel ou collectif de Hans-Dieter Bahr, Peter Berz, Bernhard Dotzler, Jochen Hörisch, Erich Hörl, Friedrich Kittler, Claus Pias, Wolfgang Pircher ou Bernhard Siegert n'a été traduit en français. Mais l'ouvrage fondateur de Ernst Kapp (1808-1896)1, que Canguilhem savait apprécier, vient de paraître à Paris, 130 ans après sa publication initiale. Il faut s'en réjouir, bien sûr, mais non sans craindre devoir longtemps attendre qu'en France l'état de l'art outre-Rhin soit suffisamment connu, ni percevoir le péril des contrebandes intellectuelles qu'une telle disparité entre les langues ne peut manquer de favoriser.

C'est toutefois aujourd'hui en histoire de l'art-tant l'art et la technique ont toujours entretenu des rapports subtils et tant les nouvelles technologies sont maintenant partout présentes dans les expositions - qu'on ignore le moins ces nouveaux développements. On en retrouve aussi des traces sous l'égide d'une médiologie par trop mondaine qui, en France, fait écran à l'essor international des Media studies, domaine dont il faudra bien que l'histoire, les sciences sociales et l'épistémologie l'investissent sérieusement, suivant des recommandations semblables à celles d'un Lucien Febvre à propos du livre et de la radio aux temps de l'Encyclopédie française. Technique, économie et information: les recherches des dernières décennies se sont déplacées entre ces trois pôles. Il appartient sans doute au renouveau actuel de l'histoire économique et de l'histoire des techniques en langue française de reconstruire sur ces trois points d'appui, et non pas seulement sur deux d'entre eux'.

Il est donc temps de faire le point. Chacun de nous, à commencer par la Revue ellemême, est pour ainsi dire traversé par les renouvellements technologiques en cours. Il importe donc de combattre sans répit les routines de l'esprit et les dédains irréfléchis alors même que les nouveaux entrants sur la scène des sciences sociales, de la philosophie ou de l'histoire des sciences et des techniques veulent prendre au sérieux et traiter par des voies nouvelles les questions que soulèvent ces mutations. Espérons donc un débat entre des spécialistes qui aujourd'hui s'expriment dans des espaces linguistiques malheureusement segmentés, débat que, bien sûr, la Revue accueillerait volontiers dans ses colonnes mais dont elle n'entendrait pas pour autant tenir l'exclusivité.

C'est à Wolfgang Pircher, lui-même protagoniste de cette scène de langue allemande, que nous avons demandé de tracer un premier tableau. Il nous a paru absurde

1. KAPP, 2007.

2. Ce renouveau est marqué notamment par StanZIANI, 2005; MARGAIRAZ et MiNARD, 2006; PÉrEz, 2008 ou encore la nouvelle série des Documents pour l'histoire des techniques que publie le CDHTE du CNAM à Paris ( $n^{\circ} 16$, paru à l'automne 2008). 
de publier son texte écrit dans une langue pesée au trébuchet dans une autre, arbitrairement choisie et coupée de ses références implicites. Cela n'en aurait donné qu'une fausse approximation tant serait demeuré sous le boisseau l'écart entre l'original et la tentative de restitution. Dans l'esprit que nous manifestions en ouvrant la nouvelle série de la Revue, voici d'une part le propos rendu dans ses grandes lignes en français et d'autre part le texte original en allemand dont on pourra apprécier à la fois la densité et l'ironie.

Pircher évoque tout d'abord «les temps anciens » de la philosophie de la technique. Le géographe Kapp entendait avoir établi qu' "inconsciemment " l'homme transmet « la forme, la relation fonctionnelle et le rapport normal » qu'il entretient avec sa propre production, ne prenant conscience de l'analogie de leur relation que dans un second temps. De là une théorie de l'Organprojektion propre au jeu entre organisme (humain) et mécanisme (artificiel). Si Kapp a emprunté le terme "inconsciemment" au médecin et philosophe naturaliste Carl Gustav Carus (1789-1869), c'est, après les travaux théoriques du mécanologue berlinois Franz Reuleaux (1829-1905), pour mettre en évidence qu'importe une "métaphysique de la machine ». Cette théorie de la projection devint dès lors un socle pour les entreprises philosophiques suivantes.

Ainsi Ernst Cassirer, qui a accordé à la technique une importance primordiale quant à ses effets sur la culture et à la philosophie la mission d'être la conscience logique de la culture, s'est-il parfois appuyé de manière critique sur la théorie de Kapp, tout en renvoyant à Marx et à son principe d'émancipation à l'égard des limites organiques. La technique gagne dès lors une autonomie qui par suite gouverne l'organisme. Et Cassirer a mis en garde celui qui dédaignerait la technique moderne en écrivant que c'est toute la culture intellectuelle qui devrait être condamnée pour autant qu'un tel jugement doive s'abattre sur la technique.

Avec les "temps d'inquiétude », Pircher désigne en premier lieu l'École de Francfort pour laquelle la culture est essentiellement la marque de l'esprit du capitalisme. La technique, par suite apparaît comme une "idéologie ». La construction technico-scientifique est le masque du politique (Marcuse). Là où l'on avait cherché une libération des forces productives, on trouve maintenant un mode de conservation des formes traditionnelles de domination, plus durable et plus étendu, quitte à espérer une science et une technique alternative, pour ainsi dire en dialogue avec la Nature (Habermas)... « Le potentiel émancipateur du travail ayant été consommé, le salut ne pouvait provenir que de l'action communicationelle. La technique a dès lors quitté la scène »conclut Pircher.

La conception de Habermas a été critiqué par Hans-Dieter Bahr qui a posé par d'autres voies la question de l'historicisation de la technique: le geste (instrumentalisation de l'organe) précède l'outil et l'usage de l'outil réalise une technique finalisée. Si bien que Bahr dissout «la technique » en une multitude de formes prises par l'emploi d'outils et de machines. Faite d'études de cas minutieuses et, à l'occasion, traversée de réminiscences de l'Organprojektion de Kapp, l'œuvre de Bahr offre une ouverture sans précédent vers le monde des outils et des machines à la philosophie 
qui l'a précédée. Du côté de la philosophie universitaire, le succès de l'époque est à mettre au compte des deux conférences où Martin Heidegger a cherché à désigner le lieu du danger technique, textes qu'on a tant interprété depuis, que Pircher renonce à en dire plus ${ }^{4}$.

Friedrich Kittler et son ouvre marquent le début de "temps plus neufs ». Résolument philosophe, Kittler tente d'allier Shannon, Turing, Foucault et Lacan. Conservateur déclaré, peu attentif aux changements sociaux et aux vertus émancipatrices de la théorie, il se soucie d'abord d'ouvrir les Lettres à ce qu'elles doivent aux sciences et aux techniques. La technique ici, selon une trajectoire comparable à celle qu'elle a connue chez Habermas, quitte l'économie pour rejoindre la communication, bien que cette fois l'attention ne porte plus sur la victoire d'une raison instrumentale mais effectivement sur la rationalité technique rendue à elle-même. Les réseaux discursifs de Kittler sont avant tout autre chose des réseaux techniques réglés sur la théorie de l'information de Shannon. Ils tiennent le discours dans ses filets, rompant-c'est le point de rencontre avec Foucault - avec les représentations spontanées de l'écriture, effet qui a produit son lot de fascination.

Dans la suite de Kittler, il faut citer les travaux sur la poste de Bernhard Siegert qui visent l'analyse du "rapport des deux fonctions essentielles que remplissent les médias: stocker (speichern) et transmettre (übertragen) ». De là une prédilection pour les relais, à tous les sens du mot, y compris ceux des centraux téléphoniques où en leur temps, les jeunes femmes standardistes apparaissaient développer une maladie énigmatique - alors dûment qualifiée - aujourd'hui saisie sur la trame des réseaux narratifs à la Kittler. À l'occasion de son habilitation (publiée en 2003), Siegert s'est rapproché de l'histoire des sciences en prenant pour objet les signes scientifiques, non du point de vue d'une sémiologie mais de celui des techniques culturelles d'écriture, de lecture, de tracé et de calcul. Il a notamment analysé les machines de papier de l'administration coloniale espagnole en Amérique et la structure électrique des laboratoires actuels.

Du signal électrique, on en vient au numérique. La belle notion de " machines de papier " apparaît à nouveau en tête d'un autre travail important où l'enquête sur les liens entre la technique et la littérature se concentre sur les notions de "communication » et de "contrôle ». Il s'agit du livre de Bernhard Dotzler. Celui-ci, excellent connaisseur de l'histoire des techniques et des théories du calcul artificiel a aussi

4. Pircher évoque d'une part la conférence "La question de la technique » parue précocement en français (HEIDEGGER, 1958) où le philosophe précisait que "la technique n'est pas ce qui est dangereux. Il n'y a rien de démoniaque dans la technique, mais il y a le mystère de son essence. C'est l'essence de la technique qui est le danger » et d'autre part le revirement - die Kehre, le tournant historial - qui a conduit Heidegger à désigner la fin de la métaphysique occidentale et son aboutissement dans le nihilisme dont la cybernétique aurait marqué l'avènement.

5. Le mot «contrôle» en français est ambigu. Par l'étymologie, il renvoie à l'inscription sur les rôles, sur les registres. Le contrôle, c'est à proprement parler la vérification de la conformité de choses et d'enregistrements. En contrôlant les papiers d'une personne, la police a besoin des deux: de la personne et des papiers. Mais depuis le milieu du XX siècle et sous l'influence de l'anglais, le mot désigne la direction, la commande voire la programmation. Sur cette palette sémantique varient bien des conditions techniques d'inscription, de vérification et de mobilisation de ce qui est contrôlé. 
publié une enquête sur la généalogie de longue durée de la machine de Turing. Peter Berz, lui-même formé par Kittler, en étudiant la mitrailleuse standard allemande de la Première Guerre mondiale est remonté jusqu'au XVIII siècle pour reconstituer une histoire des conditions de possibilité de la production en grande série des marchandises industrielles. C'est l'occasion de voir réapparaître - mais dans le corpus analysé cette fois - le mécanologue Franz Reuleaux ou encore les travaux de balistique photographique de Ernst Mach, dans les années 1880, entre autres cadavres dans le placard de la philosophie des sciences et des techniques.

Mais voici maintenant le retour du cauchemar de Heidegger - la cybernétiqueavec l'analyse qu'en a donné Erich Hörl dans sa thèse (parue en 2005) et l'intense activité de réédition dont témoignent récemment les travaux de Claus Pias ${ }^{6}$, et les recherches qui en ont procédé. L'omniprésence de l'ordinateur dans les technologies actuelles des médias explique sans doute ce regain d'intérêt pour la formation de la pensée et de la technique propres aux sciences de l'information. Le fait est qu'aujourd'hui Pias et Hörl enseignent la philosophie des médias numériques ou la théorie de médias à Vienne et à Bochum, amplifiant le mouvement déjà amorcé depuis Friedrich Kittler.

\section{ALTE ZEITEN}

Als Erfinder der Technikphilosophie in Deutschland kann der Geograph Ernst Kapp gelten. 1877 behauptet er, „durch unbestreitbare Thatsachen nachgewiesen“ zu haben, „dass der Mensch unbewusst Form, Functionsbeziehung und Normalverhältnis seiner leiblichen Gliederung auf die Werke seiner Hand überträgt und dass er dieser ihrer analogen Beziehungen zu ihm selbst erst hinterher sich bewusst wird" . Er entwirft im Zusammenspiel von Organismus und Mechanismus eine Theorie, die er unter das Stichwort der „Organprojektion“ bringt. Den Term „unbewusst“ borgte Kapp bei C. G. Carus, einem romantischen Malerfreund von Caspar David Friedrich, Arzt und Naturphilosophen. Technisch orientierte er sich an der kinematischen Theorie des Berliner Maschinenbauprofessors Franz Reuleaux, bei dem Kapp wiederum das „Unbewusste“ entdeckt und deswegen seine Analyse eine „Metaphysik der Maschine“" nennt ${ }^{2}$, worüber dieser nicht gerade glücklich war. Diese Organprojektionstheorie wurde zu einem festen Bestandteil der folgenden technikphilosophischen Unternehmungen.

Ernst Cassirer, welcher der Technik hinsichtlich ihrer Bedeutung und Wirkung für die Kultur den obersten Rang zugesteht, und der der Philosophie die Mission

6. Claus Pias a donné une édition critique des conférences interdisciplinaires organisées à New York entre 1946 et 1953 par la Fondation Macy. Elles visaient une science générale du fonctionnement de l'esprit. La cybernétique, les sciences cognitives et les sciences de l'information y ont connu leurs premières ébauches (PIAS, Hg., 2003 et 2004).

1. KAPP, 1877. Schon in einem zuvor erschienenen Buch (KAPP, 1868), ist er im Teil „Culturgeographie" auf technische Fragen eingegangen.

2. KaPp, 1877, S. 186. Franz Reuleaux galt als Philosoph unter den Maschinenbauern. [Franz Reuleaux était considéré comme philosophe parmi les constructeurs de machines.] 
zuschreibt, das logische Gewissen der Kultur zu sein, mutet ihr dementsprechend die Aufgabe zu nach den Bedingungen der Möglichkeit des technischen Wirkens und der technischen Gestaltung fragen zu müssen ${ }^{3}$. Auch Cassirer knüpft gelegentlich kritisch an die Kapp'sche Organprojektionstheorie an, indem er auf ein Gesetz der eigentlich technischen Betätigung verweist, „das man mit Karl Marx als das Gesetz der ,Emanzipation von der organischen Schranke' bezeichnet hat" ${ }^{\text {“4 }}$. Die Technik gewinnt dabei eine Autonomie, die nun selbst dem Organismus vorschreibt, wie er zu handeln hat, so erzwingt sich z.B. mit der Einführung der Nähmaschine eine neue Weise des Nähens. Schließlich schreibt Cassirer jenen, die sich von der modernen Technik abwenden, ins Stammbuch, „daß in das Verdammungsurteil, das sie über die Technik fällen, die gesamte geistige Kultur mit einbezogen werden müsste ${ }^{\text {"5 }}$.

\section{UNRUHIGE ZEITEN}

Den Philosophen und Soziologen des Frankfurter Instituts für Sozialforschung war das durchaus geläufig, denn sie identifizierten die Kultur als wesentlich vom Geist des Kapitalismus geprägt und dementsprechend war die Technik eine „Ideologie“. Der dazu wichtigste Aufsatz von Jürgen Habermas von 1968 kreist um Max Webers Begriff der „Rationalität“. Das ist auch Herbert Marcuses Ausgangspunkt der Analyse technischen Handelns als zweckrationales, dem sich Habermas einleitend widmet. Dieses Handeln macht seinen politischen Inhalt unkenntlich, d.h. nimmt ihn in das Funktionieren der technisch-wissenschaftlichen Konstruktion zurück. Nicht mehr um Befreiung qua Entfesselung der Produktivkräfte geht es nunmehr, sondern um die Erhaltung der überkommenen (kapitalistischen) Herrschaft mittels Beherrschung der Produktivkräfte. Herrschaft verewigt und erweitert sich als Technologie, woraus sich der Schluß ergibt, dass eine befreite, d.h. herrschaftsfrei kommunizierende Gesellschaft auch eine andere Wissenschaft und Technik braucht, die gleichsam in einen Dialog mit der Natur tritt statt sie zu überwältigen. Es ginge also, so Habermas, um nichts weniger als die „Resurrektion der gefallenen Natur", ein Topos der idealistischen deutschen Philosophie der in reflektierter Form auch die geheimen Hoffnungen der Frankfurter Schule lenkt ${ }^{6}$.

Habermas verwirft den Gedanken an eine andere Technik mit Hinweis auf Arnold Gehlens Anthropologie der Technik, welche technische Entwicklung als Organverstärkung zum (unbewussten) Projekt der menschlichen Gattung macht. Damit ist jedoch jede Historisierung der Technik zugunsten einer eindimensionalen anthropologischen Entwicklung sistiert. Habermas wendet sich an dieser Stelle erneut Webers Begriff der Rationalität zu, um die „Doppelfunktion des wissenschaftlich-technischen Fortschritts (als Produktivkraft und Ideologie) zu erörtern" "7. Daraus gewinnt er die Unterscheidung in Arbeit (Technik, zweckrationales Handeln) und Interaktion (kommunikatives

3. „Form und Technik“ (1930), in CASSIRER, 1995a, S. 43 f. [soit CASSIRER, 1995b].

4. Ebd., S. 73.

5. Ebd., S. 77.

6. „Technik und Wissenschaft als ,Ideologie"“, in HabERMAS, 1968, S. $54 \mathrm{f}$.

7. Ebd., S. 60. 
Handeln). Da das emanzipatorische Potential der Arbeit aufgezehrt ist, kann alles Heil nur mehr im kommunikativen Handeln gefunden werden. Damit scheidet die Technik aus der weiteren Betrachtung aus.

Während hier der gesellschaftskritische, genauer: kapitalismuskritische Zugang der Technik selbst nur eine abgeleitete Position zuwies, sie überhaupt nur als verschwommene Ganzheit auffaßte - die Technik gibt es nicht, wie man sagen könnte - und somit keinen Zugang zu den konkreten Formen technischen Wissens und technischer Praxis gestattete, versuchte Hans-Dieter Bahr die Technik sowohl im Bereich der Arbeit zu verorten, wie ihr emanzipatorisches Potential zu retten. Bahr weist in seiner Kritik der Habermaschen Analyse den Rekurs auf Gehlen (der noch dazu von Habermas verkürzt rezipiert wurde) zurück, um erneut eine Historisierung der Technik zu ermöglichen. So betont er z.B. dass das Organ selbst schon instrumentell begriffen werden muß, bevor es seine Verstärkung auslösen kann, was eben durch Arbeit geschieht. Bevor die Hand zum Hammer mutiert, muß sie schon diese Funktion zugewiesen bekommen haben: „im Gebrauch erst realisiert sich Technik als Zweck. ${ }^{8 ،}$

Es ist demnach ganz konsequent, daß Bahr in der Folge den einheitlichen Komplex „Technik“ auflöste in eine Vielzahl von Formen des Werkzeug- und Maschinengebrauchs ${ }^{9}$. Unter zwangloser Aufbietung der Philosophiegeschichte greift er das Thema in vielen Dimensionen auf, was um so besser gelingt, als es sich nicht um ein typisch akademisches Werk handelt, sondern die Theorie wird über die engeren Grenzen der Philosophie und Technikgeschichte hinaus entfaltet und bedient sich des Mythos ebenso wie der Dichtung, der alten Maschinenbücher wie der Kybernetik. An minutiös abgehandelten Fallbeispielen, z.B. der Mühle als Urform aller Maschinerie, wird dem Konstruktiven und seinen historischen Wandlungen eine gesellschaftstheoretische Position abgewonnen. Die Theorie der Organprojektion wird im Kapitel „Zuwürfe und Abbildungen. Das Technische als Projektionen des Leibes“" aufgenommen und am Beispiel von Krüppel und Superman, Vergrößerungsspiegel und Doppelgänger, sowie l'homme machine/machine vivante ${ }^{10}$ entfaltet. Kurzum, auf den 500 Seiten des Buches wird eine Welt der Werkzeuge und Maschinen eröffnet, wie sie die Philosophie bis dahin nicht kannte. Gleichwohl endet das Buch mit der Vorgeschichte seines Mißerfolgs ${ }^{11}$.

Der zweifellos erfolgreichste Text zur Technikphilosophie in Deutschland stammt von Martin Heidegger. Unter dem Titel „Die Technik und die Kehre“ versammeln sich zwei Vorträge, die 1949-1950 sowie 1955 gehalten wurden und die Heidegger zum festen Bezugspunkt der akademisch betriebenen Technikphilosophie werden ließen ${ }^{12}$. Im ersten Vortrag - „Die Frage nach der Technik“ - wird eingangs zwischen der Technik und dem Wesen der Technik unterschieden und dieses im Entbergen gefunden. Dessen Geschick wiederum ist die Gefahr. Über diese wiederum entbirgt sich das Wesen der

8. BAHR, 1970.

9. BAHR, 1983.

10. [En français dans le texte. NdR.]

11. „Dreiundzwanzig deutsche Verlage lehnten seine Veröffentlichung ab, wie es meist hieß: aus programmtechnischen Gründen ..." (Ebd., S. 498) ["Vingt-trois maisons d'édition allemandes refusèrent sa publication, arguant le plus souvent de raisons de programmation technique... ».]

12. Heidegger, 1962. 
Technik als Gestell. „Das Gestell west als die Gefahr“, heiß es im zweiten Vortrag, übertitelt „Die Kehre“"13. Die Unzahl von Interpretationen, die diese Texte erfahren haben, macht es überflüssig hier mehr zu sagen.

\section{NEUERE ZEITEN}

Während der Jahre, da Heidegger noch durch die Gänge der Universität Freiburg i.Br. schlurfte, begann dort Friedrich Kittler 1963 Germanistik, Romanistik und Philosophie zu studieren. Während bei Bahr die Philosophie gleichsam die Technik unter ihre Fittiche nimmt und unbestreitbar die Dominanz behält, kehrt sich das nun um. Der aus der Philosophie - trotz Heideggers Nähe - in die Literaturwissenschaften geflüchtete Kittler wird der (akademischen) Philosophie nur mehr einen geringen Raum gönnen ${ }^{14}$. An ihre Stelle treten Foucault und Lacan, sowie Nachrichtentechniker wie Shannon und Mathematiker wie Turing. Zugleich mit der Philosophie verliert sich auch der emanzipatorische Anspruch der Theorie. Kittler, als erklärter Konservativer, hat kein Interesse an Gesellschaftsveränderung, sondern allenfalls an einer Öffnung der Literaturwissenschaften auf ihren technisch und wissenschaftlichen Gehalt. Dementsprechend ist es auch nicht mehr die mit der Arbeit verbundene Technik, die im Mittelpunkt steht, sondern Kommunikationstechniken, also Techniken der Zirkulation, werden zu bevorzugten Themengebieten. Eine solche Wendung hatte sich ja schon bei Habermas angekündigt. Aber anders als bei Habermas geht es nun nicht mehr um eine Überwindung der instrumentellen Vernunft, wie die spezifisch technische Rationalität genannt wurde, sondern genau um diese selbst.

Ein sehr launiges Beispiel ist der Aufsatz von 1982, den Kittler an Lacan adressiert und der am Beispiel von Bram Stoker's Dracula vorführt, wie ein kleiner Medienverbund, gebildet aus handschriftlichem Tagebuch, Phonographen, Schreibmaschinen, Leichenbefunden und Zeitungsberichten zum Netz wird, in dem Graf Dracula sich verfangen muß ${ }^{15}$. Der Phonograph und seine technischen Abkömmlinge, sowie die von den Händen unverheirateter Frauen bedienten Schreibmaschinen behaupten denn auch einen prominenten Platz in Kittlers bald folgenden Büchern Grammophon, Film, Typewriter (1986) ${ }^{16}$ und Aufschreibesysteme 1800/1900 (1985) ${ }^{17}$,

13. Ebd., S. 37.

14. Das heißt keineswegs die Philosophie zu verwerfen, wie Kittler in einem Interview denn auch sagt: „In recent years I have come to think of myself simply as a philosopher who, nevertheless, is keenly interested in the reality of things, as opposed to a philosopher who reflects on reflection, as it were" (ARmitage, 2006, hier S. 18; das ganze Heft ist übrigens Kittler gewidmet.) [C'est-à-dire ne rejeter en rien la philosophie, comme le précise Kittler dans un entretien récent: "Ces dernières années, $j$ 'en suis arrivé à me considérer simplement comme un philosophe tout de même profondément intéressé par la réalité des choses, par opposition à un philosophe qui réfléchirait sur la réflexion, comme cela aurait pu être le cas. " (L'ensemble du volume où est paru l'entretien est consacré à Kittler).]

15. KitTLER, 1982.

16. KITTLER, 1986.

17. Kittler, 1985. [Précisons que si le titre allemand, Aufschreibesysteme, évoque les systèmes de notations, le titre anglais, Discourse Networks, indique des réseaux discursifs. NdR.] 
letzteres Buch ist Kittler's Habilitationsschrift in Freiburg ${ }^{18}$. Seine Worte charakterisieren dieses ungewöhnliche Buch am besten: „The concept of discourse networks is essentially a free application of Claude E. Shannon's information theory. Hence Shannon's theory, founded on information source, information channel and information receiver, that is, on informational inputs, transmission and outputs, is the engineering or technical model behind my literary experiment. Discourse Networks, 1800/1900 is, however, also deeply influenced by Michel Foucault ${ }^{19}$.“

Kittlers Schriften sind in einer deutlichen Spannung zum üblichen akademischen Kanon gehalten, sowohl was die Themen und den Stil betrifft. Sind die thematischen Zugänge bisweilen überraschend, so ist die Art des Schreibens berückend für die einen, empörend für die anderen. Das macht, daß Kittler durchwegs kontrovers beurteilt wird, aber zugleich wird damit auch die Anregung erklärbar, die er auf viele jüngere Wissenschafterinnen und Wissenschafter ausübt. Man kann vielleicht nicht gerade von einer Schule sprechen, die er begründete, auf alle Fälle aber - um im nachrichtentechnischen Jargon zu sprechen - von einem Schwingkreis mit beträchtlicher Resonanzbreite. Auch hier gilt, was die Herausgeber der Festschrift zum 60. Geburtstag in ihrem Vorwort sagen, dass es nämlich unsinnig wäre in wenigen Worten „das bisherige Werk Friedrich Kittlers, das sich von der Literaturwissenschaft über die Mediengeschichte und die Computerwissenschaft bis hin zur Mathematik, Kulturwissenschaft und Gräzistik erstreckt, auch nur im Ansatz angemessen zu würdigen. Aber man wird nicht zuviel sagen, wenn man festhält, dass keine der Disziplinen, in denen Friedrich Kittler im Laufe der letzten Jahrzehnte gearbeitet hat, nicht durch seinen Einfluß verändert worden wäre ${ }^{20 ،}$. Das läßt sich getrost auch für die Technikphilosophie behaupten.

18. In einem Interview erklärt er sein Interesse an der Schreibmaschinen-Geschichte so: ,What captivated me about the typewriter was not that there exist several high-quality monographs on the history of this technology. Rather, it was that what shows up so totally clearly from these monographs is that the introduction of young unmarried women into the production of discourses was an immediate consequence of the invention of the typewriter at the end of the 19th century. [...] Hence despite my colleagues' talk of the importance of the socio-historical conditions of the production of literature, I was the only one who considered the history of the typewriter" (ARMITAGE, 2006, S. 32). [Au cours du même entretien, il a expliqué en ces termes son intérêt pour l'histoire des machines à écrire: "Ce qui m'a captivé à propos de la machine à écrire n'était pas qu'on disposait déjà de plusieurs monographies de grande qualité sur l'histoire de cette technologie. C'était plutôt que ces monographies montraient très clairement que l'introduction de jeunes femmes célibataires dans la production des discours était une conséquence immédiate de l'invention de la machine à écrire à la fin du XIX siècle. [...] Par suite, malgré ce qu'ont pu dire mes collègues sur l'importance des conditions socio-historiques de la production de cette littérature, j'étais le seul qui a traité l'histoire de la machine à écrire. »]

19. Ebd., S. 19. ["Le concept de Aufschreibesysteme est pour l'essentiel une application libre de la théorie de l'information de Claude E. Shannon. En effet la théorie de Shannon, fondée sur l'émission de l'information, son canal et son récepteur, c'est-à-dire sur les inputs informationnels, la transmission et les outputs est l'ingénierie ou le modèle technique qui se trouve derrière mon expérimentation littéraire. Mais Discourse Networks, 1800/1900 est de même profondément influencé par Michel Foucault. »]

20. Berz, Bitsch und Siegert, Hrsg., 2003, S. 11. 
DIE FOLGEN

Bernhard Siegert, der bei Kittler in Bochum mit einer Arbeit über Kafkas postalische Strategien und Taktiken seinen ersten akademischen Grad erworben hatte, führte das Thema der Post in seiner Dissertation unter dem Titel Relais ${ }^{21}$ weiter aus. Thema des Buches ist weniger eine philosophische Reflexion des philosophischen Diskurses über Post, als vielmehr ,das Verhältnis der beiden grundlegenden Funktionen, die Medien erfüllen: Speichern und Übertragen“"22. Die Funktion der Übertragung wird im 19. Jahrhundert bekanntlich von Telegraph und Telephon erfüllt, denen ein entsprechender Raum in Siegert's Darstellung gewährt wird. Diese modernen medialen Techniken, die sich nicht mehr der Schrift, sondern des elektrischen Signals bedienen, ersetzen dementsprechend die alte Poststation durch ein technisches Bauelement, das Relais.

Die frühe Form der telephonischen Vermittlung mittels sogenannter Klappenschränke, führt denn auch wieder zur Psychoanalyse. Nicht nur befand sich in der Berggasse, unweit der Freudschen Wohnung ein derartiges Vermittlungsamt, auch eine berufsbedingte psychosomatische Störung nahm Kontakt mit dem Meister auf. Der kurze Umweg geht über die in den Vermittlungsämtern tätigen Frauen, die bisweilen von einer rätselhaften Krankheitsform befallen wurden, die man Telephon-Hysterie nannte ${ }^{23}$. Solche Verschaltungen könnte man mit Kittler ein Nachrichtennetzwerk der Jahrhundertwende nennen ${ }^{24}$.

Einen Brückenschlag zur Wissenschaftsgeschichte schließlich vollzieht die Habilitationsschrift von Siegert ${ }^{25}$. Unter Zeichenpraktiken der Wissenschaften wird hier keine Semiotik verstanden, sondern es werden Kulturtechniken des Schreibens, Lesens, Zeichnens und Zählens behandelt. „Zeichen sind keine idealen Objekte, sondern ausgedehnte Dinge; sie gehören zur Welt der res extensa und nehmen deswegen einen Platz ein. Die Welt des Symbolischen ist die Welt der Maschinen - wobei diese auch aus Papier sein können ${ }^{26}$.“ Und schließlich sind Zeichenpraktiken „mit jeweils besonderen institutionell definierten Räumen verknüpft, bestimmten ,Semiotopen', wie man sagen könnte: das Büro, das Schiff, das Atelier, das Labor, die Akademie usw ${ }^{276}$. Während im ersten Teil der Arbeit am Beispiel der administrativen Erschließung der amerikanischen Kolonien durch Spanien solche Papiermaschinen vorgeführt werden, widmet sich der zweite Teil dem Labor, in welchem die Elektrizität in all ihren Formen die Hauptrolle spielt. Damit treten wir ins Universum der elektrischen Signalverarbeitung ein, dort wo schließlich die Passage ins Digitale vollzogen wird.

21. SIEGERT, 1993.

22. Ebd., S. 16.

23. SIEGERT, 1990.

24. KITTLER, 1984

25. SIEGERT, 2003.

26. Ebd. ["Les signes ne sont pas des objets idéaux, mais des choses à considérer dans leur étendue; ils appartiennent au monde de la res extensa et y prennent place. Le monde du symbolique est le monde des machines - étant entendu que ces machines peuvent très bien être de papier.»]

27. Ebd., S. 14. [Les pratiques propres aux signes « sont liées entre elles dans des institutions particulières établies comme telles, des "sémiotopes” déterminés pour ainsi dire: le bureau, le bateau, l'atelier, le laboratoire, l'académie, etc. »] 
Der etwas exquisite Begriff „Papiermaschinen“ taucht als Titelwort in einer anderen gewichtigen Arbeit auf, die Technik und Literatur über die Begriffe von Communication und Control zu verbinden sucht ${ }^{28}$. Bernhard Dotzler bietet eine weit ausgreifende Genealogie der Turing Maschine, die bei Leibniz und den ersten Entwürfen einer Rechenmaschine von Schickard einsetzt und sie im Rahmen der Entwicklung des algorithmischen Denkens weiterführt. Dieses Interesse für das mechanische Rechnen dokumentiert sich auch in den Textsammlungen, die Dotzler entweder allein oder mit anderen herausgab : über Charles Babbage, Norbert Wiener und Alan Turing ${ }^{29}$. Babbage und Turing erscheinen auch wieder in einer neueren Arbeit, wo das Thema der Rechenmaschinen ergänzt wird um mediale Techniken ${ }^{30}$.

Schließlich ist auch noch ein Aufsatzband wichtig zu erwähnen, der Schriften von Claude Shannon enthält ${ }^{31}$. Ein Mitarbeiter an diesem Band, Peter Berz, verfaßte in rund zehnjähriger Arbeit bei Friedrich Kittler eine Dissertation, die unter dem Titel 08/15 erschien $^{32}$. Gemeint ist das deutsche Standard-Maschinengewehr des Ersten Weltkriegs. In diesem Buch wird die Geschichte der industriellen Standardisierung entfaltet, beginnend mit Honoré Blanc's neuartiger Herstellung von Infanterieschlössern in den $1780^{\text {er }}$ Jahren. Es geht um nichts weniger, als um die Bedingung der Möglichkeit von Massenfabrikation industrieller Güter. Berz zeigt mit einer atemberaubenden Materialfülle, welche konzeptuellen Leistungen in dieses Unternehmen eingingen, an dem schließlich nicht nur Maschinenbauprofessoren wie Franz Reuleaux beteiligt waren, sondern auch Physiker wie Hermann von Helmholtz und Laboratoriumsvorsteher wie Geheimrat Carl Cranz in Charlottenburg. In seinem Laboratorium wird der Kinematograph zur Aufzeichnung der Geschoßbewegungen benutzt und entsprechend den besonderen Anforderungen weiterentwickelt. „Mit dem Kinematographen aus Charlottenburg aber läßt sich nicht nur die Geschwindigkeit fliegender Geschosse vermessen. Er steigt schnell zum Leitmedium für die Gesamtheit des ballistischen Feldes und aller seiner Bewegungserscheinungen auf ${ }^{33}$ " "Damit kommen auch die ballistisch-photographischen Versuche, die Ernst Mach und Peter Salcher in den 1880 ${ }^{\text {er }}$ Jahren durchführen auf einen medientechnisch hohen Standard. Diese wiederum sind Thema eines Buches, in dem die entsprechenden Notizbücher von Ernst Mach abgedruckt und kommentiert, wie auch eine Reihe von Aufsätzen zum Thema versammelt $\operatorname{sind}^{34}$. Dies als ein Beispiel für den zwanglosen Übergang der Technikphilosophie und -geschichte in die Wissenschaftstheorie und -geschichte. Daran ließen sich viele andere Beispiele anfügen, zumal die Wissenschaftsgeschichte den technischen Aspekt der neuzeitlichen Naturwissenschaften entdeckte, die als wesentlich quantifizierende einen zunehmenden Park an Instrumenten benötigen ${ }^{35}$.

\footnotetext{
28. DotZler, 1996a.

29. Dotzler und Kittler, Hrsg., 1987; Dotzler, Hrsg., 1996b, 2002.

30. DotZLER, 2006.

31. Kittler et al., Hrsg., 2000.

32. BERZ, 2001.

33. Ebd., S. 484.

34. Hoffmann und Berz, Hrsg., 2001.

35. Dazu neuerdings Hoffmann, 2006.
} 


\section{NOCH EINMAL HEIDEGGER}

Martin Heidegger hatte 1966 (publiziert im Todesjahr 1976) in einem Interview mit dem Nachrichtenmagazin „Der Spiegel“ die Behauptung, Vermutung oder Befürchtung geäußert, die Philosophie werde durch die Kybernetik ersetzt werden. Erich Hörl, der an der Humboldt-Universität Berlin mit der Arbeit „Die heiligen Kanäle. Historischepistemologische Untersuchungen zur archaischen Illusion der Kommunikation“ bei Thomas Macho und Friedrich Kittler dissertierte, widmet sich speziell dem Verhältnis von Heideggers Philosophie mit der Kybernetik ${ }^{36}$. „Die neuen kybernetischen ,Leitvorstellungen' von ,Information, Steuerung, Rückmeldung' und insbesondere ihr Charakter als ,Modellbegriffe' galten ihm - und diese Zuspitzung ist ein, wenn nicht das Kernmoment seiner philosophischen Politik - als Ausdruck der totalen Technisierung des Denkens, die dessen überliefertes, für die Philosophie insgesamt signifikantes logischmathematisches Verständnis nunmehr in Maschinengestalt und als Maschinendenken realisierte. Mit der Kybernetik verwirklichte sich die Metaphysik in ihrer letzten Figur, dem ,Gestell', das für Heidegger die moderne Technik charakterisierte ${ }^{37}$.“

Die Erinnerung an die Geschichte der Kybernetik wird von einem bemerkenswerten Unternehmen von Claus Pias unterstützt, erneut die Protokolle der Macy-Konferenzen zugänglich zu machen ${ }^{38}$. Diese von der Macy-Foundation finanzierten Konferenzen brachten die Größen der damaligen Kybernetik zusammen, wo die Referate der Teilnehmer wie Norbert Wiener, Claude Shannon, Warren McCulloch, Ross Ashby, Heinz von Foerster und anderen vorgestellt und diskutiert wurden. In einem zweiten Band werden aktuelle Beiträge zur Kybernetik versammelt ${ }^{39}$. Hierin findet sich auch ein Essay von Erich Hörl zu besagter Befürchtung von Heidegger, die Kybernetik würde den Platz der Philosophie einnehmen ${ }^{40}$. Die Kybernetik ist ein prägnantes Beispiel dafür, wie aus einer speziellen Technik der Regelung eine Disziplin des Denkens entstehen kann, das eine gewisse Zeit interdisziplinäres Forschen überhaupt repräsentierte. Unter dem Dach der Kybernetik, wie von Norbert Wiener in seinem Gründungsakt schon vorgesehen ${ }^{41}$, versammelten sich neben den Nachrichten- und Regelungstechniken ja auch eine Reihe von Wissenschaften, von der Ökonomie, Politik über Biologie und Psychoanalyse auch Pädagogik, Philosophie und allgemeine Systemtheorien. Sich ihrer Geschichte anzunehmen, heißt nicht gleichzeitig sie wieder in Geltung bringen zu wollen, sondern stellt eher den Versuch dar, die Vorgeschichte der elektronischen Medien zu erfassen.

Pias und Hörl lehren Philosophie der digitalen Medien bzw. Medientheorie in Wien und Bochum und dies zeigt auch die generelle Verschiebung im Interesse an Technik,

36. HÖRL, 2005. Eine seiner aktuellen Forschungsprojekte trägt den Titel: „Heidegger und die Kybernetik. Zur historischen Epistemologie der, Weltfrage des Denkens' in der Wissensordnung des Kalten Krieges." [L'un de ses projets de recherche en cours a pour titre: "Heidegger et la cybernétique. Pour une épistémologie historique de la "question mondiale de la pensée" dans l'ordre du savoir de la Guerre froide. »]

37. HörL, 2008, S. 163 f.

38. Pias, Hg., 2003.

39. PiAs, Hg., 2004.

40. HÖRL, 2004.

41. WIENER, 1948. 
die schon bei Friedrich Kittler einsetzte. Insbesondere seit der Computer alle anderen Medien an sich zieht, sind Medientheorien gleichsam nur mehr unter digitalem Anspruch zu haben. Somit läßt sich berechtigterweise die Frage nach den Zukünften des Computers stellen, die auch die Frage nach den unseren ist oder einmal gewesen sein wird ${ }^{42}$.

\section{KOMMENTIERTE LITERATUR}

Armitage (John), 2006, "From Discourse Networks to Cultural Mathematics. An Interview with Friedrich A. Kittler”, Theory, Culture \& Society, vol. 23, n7-8, p. 17-38.

BAHR (Hans-Dieter), 1970, Kritik der „Politischen Technologie“. Eine Auseinandersetzung mit Herbert Marcuse und Jürgen Habermas, Frankfurt, Europäische Verlagsanstalt.

BAHR (H.-D.), 1983, Über den Umgang mit Maschinen, Tübingen, Konkursbuchverlag.

Berz (Peter), 2001, 08/15. Ein Standard des 20. Jahrhunderts, München, Fink Verlag.

Berz (Peter), Bitsch (Annette) und Siegert (Bernhard), Hrsg., 2003, FAKtisch. Festschrift für Friedrich Kittler zum 60. Geburtstag, München, Fink Verlag.

BrIAN (Éric), 2007, «Travail de synthèse et diversité des langues/Synthesis work and diversity of languages ", Revue de synthèse, t. 128, $\mathrm{n}^{\circ} 1-2$, p. 5-18.

CASSIRER (Ernst), 1995a, Symbol. Technik, Sprache. Aufsätze aus den Jahren 1927-1933, herausgegeben von Ernst Wolfgang ORтн und John Michael KroIs unter Mitwirkung von Josef M. WerLe, Hamburg, Meiner Verlag.

CAssirer (E.), 1995b, «Forme et technique », dans Id., Écrits sur l'art, édition et postface de Fabien CApeIllères, présentation de John M. Krois, textes traduits par Christian Berner, Fabien Capeillères, Jean Carro et Joël Gaubert, Paris, Cerf/Passages, p. 61-100.

Dotzler (Bernhard), 1996a, Papiermaschinen: Versuch über Communication \& Control in Literatur und Technik, Berlin, Akademie Verlag.

Dotzler (B.), Hrsg., 1996b, Babbages Rechen-Automate, Wien/New York, Springer Verlag.

Dotzler (B.), Hrsg., 2002, Norbert Wiener. Futurum exactum. Ausgewählte Schriften zur Kybernetik und Kommunikationstheorie, New York/Wien, Springer Verlag.

Dotzler (B.), 2006, Diskurs und Medium. Zur Archäologie der Computerkultur, München, Fink Verlag.

Dotzler (Bernhard) und Kittler (Friedrich), Hrsg., 1987, Alan Turing. Intelligence Service. Schriften, Berlin, Brinkmann \& Bose.

Habermas (Jürgen), 1968, Technik und Wissenschaft als „Ideologie“, Frankfurt, Suhrkamp Verlag.

Hagner (Michael) und Hörl (Erich), 2008, Die Transformation des Humanen. Beiträge zur Kulturgeschichte der Kybernetik, Frankfurt, Suhrkamp Verlag.

HeIDEgGer (Martin), 1958, « La question de la technique », dans ID., Essais et conférences, textes traduits par André PréAU, Paris, Gallimard, p. 9-48.

Heidegger (M.), 1962, Die Technik und die Kehre, Pfullingen, Neske Verlag.

HoffmanN (Christoph), 2006, Unter Beobachtung. Naturforschung in der Zeit der Sinnesapparate, Göttingen, Wallstein Verlag.

Hoffmann (Christoph) und Berz (Peter), Hrsg., 2001, Über Schall: Ernst Machs und Peter Salchers Geschoßfotografien, Göttingen, Wallstein Verlag.

42. Pias, Hg., 2005. 
Hörisch (Jochen) und Wetzel (Michael), Hrsg., 1990, Amaturen der Sinne: Literarische und technische Medien 1870 bis 1920, München, Fink Verlag.

HöRL (Erich), 2004, „Parmenideische Variationen. McCulloch, Heidegger und das kybernetische Ende der Philosophie“, in PIAS, Hg., 2004, S. 209-225.

HörL (E.), 2005, Die heiligen Kanäle: über die archaische Illusion der Kommunikation, Zürich/ Berlin, Diaphanes Verlag.

Hörl (E.), 2008, „Das kybernetische Bild des Denkens“, in HaGner und HörL, 2008, S. 163-195.

KAPP (Ernst), 1868, Vergleichende Allgemeine Erdkunde in wissenschaftlicher Darstellung, zweite verbesserte Auflage, Braunschweig, Westermann Verlag.

Kapp (E.), 1877, Grundlinien einer Philosophie der Technik. Zur Entstehungsgeschichte der Cultur aus neuen Gesichtspunkten, Braunschweig, Westermann Verlag.

Kapp (E.), 2007, Principes d'une philosophie de la technique, Paris, Vrin.

KitTLER (Friedrich), 1982, „Draculas Vermächtnis“, in ZETA 02. Mit Lacan, Berlin, Rotation Verlag, 1982, S. 103-136 (Wiederabgedruckt in KitTLER, 1993, S. 11-57).

KitTLER (F.), 1984, „Flechsig/Schreber/Freud. Ein Nachrichtennetzwerk der Jahrhundertwende“, in Der Wunderblock, Nr. 11/12, S. 56-68.

KittLer (F.), 1985, Aufschreibesysteme 1800/1900, München, Fink Verlag (2. erw. Aufl. 1987; 3. vollständig überarbeitete Aufl. 1995). [Discourse Networks 1800/1900, Stanford, Stanford University Press, $1990 ; 2^{\mathrm{e}}$ éd., 1992.]

KitTler (F.), 1986, Grammophon, Film, Typewriter, Berlin, Brinkmann \& Bose [Gramophone, Film, Typewriter, Stanford, Stanford University Press, 1999].

Kittler (F.), 1993, Draculas Vermächtnis. Technische Schriften, Leipzig, Reclam Verlag [Literature, Media, Information Systems. Essays, Amsterdam, GB Arts International, 1997; Japanische Übersetzung in Tokyo, 1998].

Kittler (Friedrich), Berz (Peter), Hauptmann (David) und Roch (Axel), Hrsg., 2000, Claude E. Shannon. Ein/Aus. Ausgewählte Schriften zur Kommunikations- und Nachrichtentheorie, Berlin, Brinkmann \& Bose.

Margairaz (Dominique) et Minard (Philippe), 2006, Le Marché et son histoire, dossier de la Revue de synthèse, $\mathrm{n}^{\circ} 2,2006, \mathrm{p} .235-378$.

PÉREZ (Liliane), 2008, La Pièce et le geste. Entreprises, cultures opératoires et marchés à Londres au XVIII ${ }^{e}$ siècle, Paris (habilitation à diriger les recherches de l'université de Paris-I).

PIAS (Claus), Hg., 2003, Cybernetics - Kybernetik. The Macy-Conferences 1946-1953. Transactions - Protokolle, Zurich/Berlin, diaphanes Verlag.

Pias (C.), Hg., 2004, Cybernetics - Kybernetik. The Macy-Conferences 1946-1953. Essays \& Dokumente, Zurich/Berlin, diaphanes Verlag.

PiAs (C.), Hg., 2005, Zukünfte des Computers, Zurich/Berlin, Diaphanes Verlag.

Siegert (Bernhard), 1990, „Das Amt des Gehorchens. Hysterie der Telephonistinnen oder Wiederkehr des Ohres 1874-1913“, in Hörisch und Wetzel, Hrsg., 1990, S. 83-106.

Siegert (B.), 1993, Relais. Geschicke der Literatur als Epoche der Post: 1751-1913, Berlin, Brinkmann \& Bose [Relays. Literature as an Epoch of the Postal System, Stanford, Stanford University Press, 1999].

SiEgert (B.), 2003, Passage des Digitalen. Zeichenpraktiken der neuzeitlichen Wissenschaften 1500-1900, Berlin, Brinkmann \& Bose.

Stanziani (Alessandro), 2005, Histoire de la qualité alimentaire. $X I X^{e}-X X^{e}$ siècle, Paris, Le Seuil.

WIENER (Norbert), 1948, Cybernetics or control and communication in the animal and the machine, Paris, Hermann (Actualités scientifiques et industrielles, $\mathrm{n}^{\circ} 1053$ ). 